\title{
Research on Presentation of Multimedia Interactive Electronic Sand
}

\author{
Table \\ Daogui Lin \\ Fujian Polytechnic of Information Technology, Fuzhou, 350003, China
}

\begin{abstract}
Multimedia interactive electronic sand table presents the virtual design site and can intuitively present the site layout, integrating multiple high technologies, such as multimedia display technology, network information transmission technology, analog-to-digital sensing technology, touch screen display technology and database technology. The intelligent function assisted program planning can be started by coordinating with the corresponding functional technology, calculating the relevant information data and conducting assisted assessment according to the expected effects. In the real estate marketing presentation, the planned picture should be presented in the virtual real landscape when the sand table design planning is completed and the used auxiliary functions include electronic three-dimensional map, electronic sand table dynamic landscape and others formed by three-dimensional modeling, experiment point change and terrain rendering of virtual real architectural space. It realizes a sense of reality and is conducive to the real estate marketing. In this paper, the presentation of multimedia interactive electronic sand table is studied.
\end{abstract}

Key words: Multimedia; electronic sand table; virtual simulation technology; computer aided design; presentation

With the development of science and technology, the multimedia interactive electronic sand table has become an important direction of informatization development. The traditional sand table shows just one-dimensional time and space, which is far from enough to present the current diversified and fast-paced living environment. Before the research and development of multimedia interactive electronic sand table, the terrain simulation technology is mainly adopted, covering three-dimensional terrain modeling and inversion, image processing and other contents. The design of real estate sand table adopts three-dimensional simulation 
technology, which can realize the multimedia electronic interaction to simulate the whole landscape of the house and then show it by the visualization system.

\section{Concept and function of sand table}

(I) Concept of sand table

Sand table is divided into simple sand table and permanent sand table. The simple sand table is composed of sand and chess on the ground for temporary use and the permanent sand table is made of high-precision, sturdy and durable materials, such as foam plastic board, gypsum powder and pulp, characterized by light weight and no deformation after long-term use. Compared with the simple sand table, the permanent sand table is a miniature of geographic area, which is durable, convenient to appreciate and of fine production.

(II) Function of sand table

Sand table can shape the virtual presentation environment. The traditional sand table of complex craftsmanship is inconvenient to transport and modify. With the development of information technology, the electronic sand table with high high-tech content emerges. The electronic sand table mainly adopts the multimedia simulation system. In the drawing of sand table, the simulation system will mobilize various functions, such as digital photo, 3-dimensional image, virtual reality (VR) and interactive interface to provide simulation terrain for uses under the command of drawer and also conduct interactive operation of virtual environment that is drawn. The electronic sand table based on the computer graphics and image technology avoids the disadvantages of traditional sand table, mainly embodied in the shaping of the virtual picture of material object. All the data including terrain information data are based on the terrain database to construct the virtual 3D terrain realistically, thus providing real operating environment for sand table users and capable of interactive operation.

\section{Building electronic sand table}

According to the needs of practical application field, the sand table can be divided into different types. In the real estate field, the building sand table is preferential, which shows the architectural art and structure by means of miniature 
material object, whether the single model and group composition and converts the specific circumstance in the building into the specific image. As the information technology is integrated into the design field and based on the three-dimensional visualization technology and geographic information system, the building electronic sand table is developed by adopting the virtual reality technology and combined with the design sketch of electronic sand table. However, due to the large design data size and scale, it is difficult technologically and unfavorable for operation if it is used for network loading. In order to improve the visual effects and application value of building sand table and bring substantial profits to the real estate sales, the real estate development company builds the building “electronic sand table” system together with the related software developer and a platform for the exchange between real estate company and users has formed.

Different from the ordinary visual experience, the building "electronic sand table” system provides dynamic visual experience and has strong three-dimensional animated expression and interaction. The client who sees the building "electronic sand table" can visit the building design and layout according to the preset route on the sand table and see the overall environmental effects from a global perspective.

In addition, the building “electronic sand table” can release more information. Its virtual reality technology can fully integrate the building with the urban landscape to form an integrated sense of beauty. The design in the building adopts three-dimensional animated navigating effects so that the visitors can see the indoor scenes clearly. In addition to the overall appearance presentation of building "electronic sand table", the virtual technology also shows the details of building design, such as entrance hall and lobby in the building, square, green field and courtyard in the community, waterscape and other greenery landscape so as to improve the real estate project planning quality.

\section{Presentation of multimedia interactive electronic sand table}

(I) The virtual presentation of multimedia electronic sand table is closer to the reality

Electronic sand table, adapting to the information age, uses advanced geographic information technology and looks for the relevant geographic information 
of each point according to the acquired real and accurate three-dimensional geographic information data. These information adopt real-time dynamic look-up method for temporary use. The electronic sand table has three-dimensional simulation function and can draw the topographic features and material objects on the three-dimensional coordinates of computer accurately, such as road, building, river and long-range plan, in addition to show the information by three-dimensional coordinate. For the efficiency, the three-dimensional single point, route and others can be assessed by computer intelligent analysis method. For example, the planning of relevant factor of community environment in the three-dimensional space should consider the geographic environment to make the virtual presentation effects closer to the reality.

(II) Multimedia electronic sand table should conform to people’s living habits

"Electronic sand table" is made by multimedia computer with touch screen integrated functions and then to present by all-around interactive form. At present, the commonly used electronic sand tables include three-dimensional electronic sand table, multimedia touch sand table and sound and light sand table. For the composition, with the controllers of various functions, such as driver, logic controller, touch controller and stage light controller, as the center, the electronic sand table presents the material objects in a projecting mode on the large screen by coordinating with the sand table model and using the multimedia presentation software. To make the electronic sand table have multimedia presentation effects, the light of sand table model and the stage light and action needing synchronous presentation should be of multimedia automatic control according to the various information in the sand table and the image, video, picture, audio and character in the multimedia should be of remote control. In case of short-distance operation, the manual mode can be adopted so as to get better presentation effects of multimedia electronic sand table.

(III) Multimedia electronic sand table realizes the intelligent interactive management

With the development of intelligent interactive technology, the intelligent management of human-virtual environment interaction is realized during the sand table design. 
The years from 2006 to 2020 are the era that the intelligent technology is used, which has been mentioned in the Outline for National Long-Term Scientific and Technological Development. In China, the intelligent sensing technology and virtual reality technology are listed as the key advanced technologies in a period of time in the future.

In China's real estate field, to improve the competitive strength of real estate marketing, the high-definition image of building can be presented by the presentation module of sand table, including the floors of building and households on each floor, which can be presented to the client in a three-dimensional way. The building environment constructed with digital information technology as the carrier applies the interactive technology to the computer sand table production integration system, thus realizing the "people-centered" systematic operation technology. During the page load, the building project diagram and number of buildings can be loaded and the floor that the client clicks will be sprung out and all the households on this floor will be presented clearly.

The electronic sand table is not a tool simply, but adopts the modern information display means and introduces the virtual interaction technology to realize the virtual environment. From the presentation of electronic sand table, it generates the real estate model by using computer software and then uses the diversified features of channel to bring people into the electronic virtual real estate planning environment. Its quick response, highly automatic command function and presentation of three-dimensional building pictures satisfy the client's needs, which can not only help marketing, but also accelerate the sales speed.

(IV) Design presentation of multimedia building electronic sand table

Corresponding to the traditional sand table model, the building electronic sand table eliminates the disadvantages of traditional sand table, such as intuitive property and lacking of flexibility, for which not only the building presentation method is changed, but also the large screen display is used to replace the fixed intuitive property of traditional sand table. The points on the sand table can be identified by multi-point touch screen. 
In the program planning, the multimedia interactive electronic sand table system is used and its intuitive property can be fully embodied by adjusting the position of sand table model according to the needs. In the program planning, the planning parameter should be calculated and the planning effects should be assessed. Each function of sand table should get the multimedia assisted supports. After program planning, it is the presentation stage. At that time, the parameters will be presented on the sand table, thus providing effective supports for the constraint verification of program planning and parameter optimization.

the sharing of electronic sand table can be realized when the multimedia computer runs under Internet. From the interactive perspective, the electronic sand table promotes the video interaction and realizes multi-point touch after processing by intelligent interactive technology.

From the composition of multimedia interactive electronic sand table, it mainly includes two parts: hardware subsystem and software subsystem. The computer hardware equipment related to the electronic sand table are large screen display, image fusion controller, multi-point interaction and capture action. The software system of electronic sand mainly has server and client. The various information data can be connected by the interface of the system. The traditional sand table is more intuitive in the drawing of geographic environment, but with low implementation efficiency mainly because it lacks of flexibility. The multimedia electronic sand table can set up correlation with various data and list the required environment unit in it to present the interactive sand table.

\section{Conclusion:}

All in all, in the real estate marketing, the traditional building electronic sand table still has application value. But with the continuous improvement of scientific and technological level, the traditional building electronic sand table develops and perfects continuously. By integrating the multimedia display technology, network information transmission technology, analog-to-digital sensing technology, touch screen display technology, database technology and other technologies, the multimedia interactive electronic sand table is designed, which is closer to the reality 
in the virtual presentation, conforms to people's living habits and realizes the intelligent interactive management. It is worthy of in-depth discussion and analysis of relevant people.

\section{Acknowledgments}

This paper was the research project of Fujian Polytechnic of Information Technology in 2013 titled Research on Presentation of Multimedia Interactive Electronic Sand Table (No.: Y13104).

\section{References:}

[1] Li Chunling. Design of Infrared Remote Control Sand Table Model System based on UM3758-108A[J]. Electronic Component \& Device Applications, 2010.12 (11).

[2] Sanyal S, Bansal M, Banerjee S, etal. Modeling of Free • Form Surfaces and Shape From Shading/Second International Symposium 011 3D Data Processing [C]. Washington, DC USA : Visualization and Transmission(3DPVT104), 2004.

[3] Sugihara K. Mathematical Structure of Line Drawings of Polyhedrons-Towards Man-machine Communication by Meansof Line Drawings [J]. IEEE. Transactions on pattern Analysis and Machine intelligence, 2005. 4(05).

[4] Han F and Zhu S C. Bayesian Reconstruction of 3D Shapes and Scenes From A Single Image [DB/OL]. www. static. CC. gatech. Edu, 2006.

[5] Zhu Jie. Development and Application of Touch-Screen Interactive Electronic Sand Table[J]. Journal of Geomatics Science and Technology, 2011.28 (04).

[6] Booth Bob. Using ArcGIS 3D Analyst [Z]. Environmental System Research Institute, Inc, 2000. 